\title{
ITO/Poly(Aniline)/Sol-Gel Glass: An Optically Transparent, pH-Responsive Substrate for Supported Lipid Bilayers
}

\author{
Ahmed Al-Obeidi, Chenhao Ge, Kristina S. Orosz, and S. Scott Saavedra \\ Department of Chemistry and Biochemistry, University of Arizona, Tucson, AZ 85721-0041, USA \\ Correspondence should be addressed to S. Scott Saavedra; saavedra@email.arizona.edu
}

Received 24 November 2012; Accepted 21 February 2013

Academic Editor: Giancarlo Righini

Copyright (C) 2013 Ahmed Al-Obeidi et al. This is an open access article distributed under the Creative Commons Attribution License, which permits unrestricted use, distribution, and reproduction in any medium, provided the original work is properly cited.

\begin{abstract}
Described here is fabrication of a $\mathrm{pH}$-sensitive, optically transparent transducer composed of a planar indium-tin oxide (ITO) electrode overcoated with a poly(aniline) (PANI) thin film and a porous sol-gel layer. Adsorption of the PANI film renders the ITO electrode sensitive to $\mathrm{pH}$, whereas the sol-gel spin-coated layer makes the upper surface compatible with fusion of phospholipid vesicles to form a planar supported lipid bilayer (PSLB). The response to changes in the $\mathrm{pH}$ of the buffer contacting the solgel/PANI/ITO electrode is pseudo-Nernstian with a slope of $52 \mathrm{mV} / \mathrm{pH}$ over a $\mathrm{pH}$ range of 4-9. Vesicle fusion forms a laterally continuous PSLB on the upper sol-gel surface that is fluid with a lateral lipid diffusion coefficient of $2.2 \mu \mathrm{m}^{2} / \mathrm{s}$ measured by fluorescence recovery after photobleaching. Due to its lateral continuity and lack of defects, the PSLB blocks the pH response of the underlying electrode to changes in the $\mathrm{pH}$ of the overlying buffer. This architecture is simpler to fabricate than previously reported ITO electrodes derivatized for PSLB formation and should be useful for optical monitoring of proton transport across supported membranes derivatized with ionophores and ion channels.
\end{abstract}

\section{Introduction}

An artificial lipid bilayer that modulates the transmembrane flux of ions to an underlying electrode is the basis for several types of bioelectronic devices, including biosensors and biomimetic fuel cells [1-6]. Modulation of ion flux across a lipid membrane can be achieved using ionophores, ion channels, and proton pumping proteins (e.g., [7-13]). To create these devices, lipid bilayer assemblies supported on a variety of electrode materials have been prepared and characterized (e.g., $[6,14-17]$ and references therein). However most commonly used electrode materials (e.g., Au, doped $\mathrm{Si}$, indium-tin oxide (ITO)) do not respond selectively to any particular ion.

Ion selectivity can be obtained by chemically modifying the electrode, and conductive polymers are promising candidates for this purpose. These polymers exhibit electrical properties characteristic of semiconductors [18], and like semiconductors, conductivity can be enhanced through doping [19-21]. The ability to adjust conductivity and charge in these polymers has led to their use as electrochemical and optical sensing elements [22, 23]. For example, doping/dedoping of poly(aniline) (PANI) can be accomplished by changing the $\mathrm{pH}$ of the surrounding medium, and this property makes PANI useful as a $\mathrm{pH}$-sensing material [24]. Both electrochemical and optical $\mathrm{pH}$ sensors based on PANI have been reported [22, 23, 25-27], which suggests the possibility of using a PANI-coated electrode as a protonselective substrate for a planar supported lipid bilayer (PSLB) $[28,29]$.

Fusion of lipid vesicles to form a continuous, extended PSLB occurs readily on hydrophilic, smooth substrates such as glass and mica [30]. For spectroelectrochemical sensing, ITO is the preferred substrate; however, the surface of ITO is too rough to support formation of a continuous PSLB that is largely free of ion-permeable defects. Adsorption of PANI can decrease the surface roughness of ITO but vesicle fusion directly on PANI generates multilayer PSLBs of uneven thickness $[25,29]$. One strategy to overcome these problems is overcoating the PANI-modified electrode with a material that promotes vesicle fusion. Recently we used this strategy to prepare PANI-modified ITO substrates [28]. PANI 
nanowires were grown electrochemically in the pores of a solgel glass layer that was spin coated on ITO. Application of a second sol-gel layer generated a smooth surface on which a PSLB was deposited by vesicle fusion, and this membrane was very effective in blocking diffusion of protons to the $\mathrm{pH}$ sensitive PANI nanowires. The drawback of this strategy is the complexity of the fabrication process.

Here we describe a much simpler approach. A thin layer of PANI is adsorbed to ITO which renders the electrode sensitive to $\mathrm{pH}$. A porous sol-gel layer is then spun cast over the PANI layer. The response to changes in the $\mathrm{pH}$ of the buffer contacting the sol-gel/PANI/ITO electrode is pseudoNernstian and reversible. The sol-gel surface is smooth and hydrophilic which supports vesicle fusion of a fluid PSLB that blocks the $\mathrm{pH}$ response due to the very low ion permeability of the membrane.

\section{Material and Methods}

2.1. Materials. The following chemicals were purchased from Aldrich and used without further purification: tetraethyl orthosilicate (TEOS; 99.999\%); triethoxymethylsilane (MTES; 99\%); polyaniline (PANI; 10,000 MW emeraldine base); anhydrous 1-methyl-2-pyrrolidinone (NMP; 99.5\%). L- $\alpha$ phosphatidylcholine (Egg, Chicken; 99\%) (egg PC) in chloroform and 1-palmitoyl-2-[6-[(7-nitro-2-1,3-benzoxadiazol4-yl)amino]hexanoyl]-sn-glycero-3-phosphocholine (NBDPC) were purchased from Avanti Polar Lipids (Alabaster, AL). Ethanol 200 proof was purchased from Decon Laboratories. Universal buffer was prepared from $11.4 \mathrm{mM}$ boric acid, $6.7 \mathrm{mM}$ citric acid, and $10.0 \mathrm{mM}$ monosodium phosphate dissolved in deionized (DI) water $(18 \mathrm{M} \Omega \cdot \mathrm{cm})$.

2.2. Electrode Preparation. Float glass slides coated with indium tin oxide (ITO; $8-12 \Omega$ per square, $120-160 \mathrm{~nm}$ thick; Delta Technology, Stillwater, MN) were cleaned as described in Supplementary Material, available online at http://dx.doi .org/10.1155/2013/676920, and stored in ethanol until use. Stored ITO electrodes were sonicated in ethanol for 15 minutes, dried under nitrogen, then oxidized in an air plasma (Harrick PDC-3XG) for 15 minutes, and then immediately immersed into $0.5 \mathrm{mg} / \mathrm{mL}$ PANI in anhydrous NMP for 30 minutes. The slides were then rinsed with deionized water and dried gently under a nitrogen stream. The sol-gel layer was prepared from $1 \mathrm{~mL}$ MTES, $0.374 \mathrm{~mL}$ TEOS, $0.794 \mathrm{~mL}$ ethanol, $0.465 \mathrm{~mL} \mathrm{DI} \mathrm{H}_{2} \mathrm{O}$, and $25 \mu \mathrm{L} 1 \mathrm{M} \mathrm{HCL}$. The solution was stirred for 3 hours, passed through a $0.2 \mu \mathrm{m}$ filter, and diluted with $2.49 \mathrm{~mL}$ ethanol. After stirring for an additional hour, the sol was spun cast $(2750 \mathrm{rpm})$ onto the PANI-coated ITO electrodes, and then cured at $50^{\circ} \mathrm{C}$ for 18 hours.

2.3. Lipid Bilayer Deposition. Egg PC was dried under argon and then under vacuum for 4 hrs. Lipids $(1 \mathrm{mg} / \mathrm{mL})$ were resuspended in $\mathrm{pH} 8$ universal buffer, vortexed, and then sonicated for 90 minutes at $35^{\circ} \mathrm{C}$ (W-380 sonicator, Ultrasonics, Inc.) to generate a clear solution of vesicles. Just prior to vesicle fusion, ITO/PANI/sol-gel electrodes were oxidized in an air plasma for 10 seconds to generate a hydrophilic surface.
Vesicle fusion was performed using procedures described previously [28] to generate a PSLB on the electrode, followed by rinsing away unfused vesicles with buffer.

2.4. Characterization. Potentiometric measurements were performed using a Denver $215 \mathrm{pH} / \mathrm{ISE}$ meter with $\pm 0.1 \mathrm{mV}$ accuracy and $6 \times 10^{12} \Omega$ input impedance. Electrodes were conditioned in $\mathrm{pH} 4$ buffer overnight before potentiometric measurements. After the $\mathrm{pH}$ of the buffer in contact with an electrode was changed, the electrode was allowed to equilibrate for 3-5 minutes before the potential was measured.

Atomic force microscopy (AFM) was performed using an Agilent 5500 (Agilent Technologies) with etched Si AFM tips (Mikromasch NSC15) under AAC mode. Images were acquired in air over a scan area of $100 \mu \mathrm{m}^{2}$. For each sample, images were acquired at several locations. RMS roughness values were obtained from $10 \mu \mathrm{m}^{2}$ regions within the $100 \mu \mathrm{m}^{2}$ scan area.

Broadband attenuated total reflection (ATR) spectroscopy of ITO/PANI/sol-gel electrodes was performed using a custom built instrument described in previous papers $[25,31]$ and in Supplementary Material. Fluorescence imaging and fluorescence recovery after photobleaching (FRAP) measurements on PSLBs deposited on ITO/PANI/sol-gel electrodes were performed as described in [28, 32] and in Supplementary Material. For FRAP, PSLBs composed of egg PC (the blank) or NBD-PC/egg PC (the sample, $0.05 / 0.95 \mathrm{~mol} / \mathrm{mol})$ ) were formed on pairs of ITO/PANI/solgel electrodes mounted in the two compartments of a liquid cell.

Water contact angle measurements were performed using a Kruss Drop Shape Analysis (DSA 10Mk2) instrument at ambient temperature and humidity. Ellipsometry on PANI and sol-gel layers deposited on ITO and oxidized Si wafers was performed at $632.8 \mathrm{~nm}$ using an incident angle of 70 degrees.

\section{Results and Discussion}

3.1. Characterization of ITO/PANI/Sol-Gel Electrodes. Solgel coatings were prepared using MTES and TEOS because, based on past work $[28,33,34]$, a mixture of these precursors provides an appropriate combination of mechanical durability and porosity. Since MTES is trifunctional, it generates a less dense, more porous film than pure TEOS and is less susceptible to cracking during drying. After curing for 18 hours at $50^{\circ} \mathrm{C}$, ITO/PANI/sol-gel electrodes were qualitatively tested to ensure that the sol-gel reaction was essentially complete by exposing slides to $30 \mathrm{mM}$ universal buffer, $\mathrm{pH}$ 4. An incomplete reaction was indicated by the layer becoming translucent after immersion. Only electrodes that did not exhibit any changes in appearance were used in further experiments.

AFM, water contact angle measurements, and ellipsometry were used to characterize surface morphology, wettability, and layer thickness. Measurements on ITO yielded thickness values of $4.2 \mathrm{~nm}( \pm 0.22, n=4)$ for the adsorbed PANI layer 


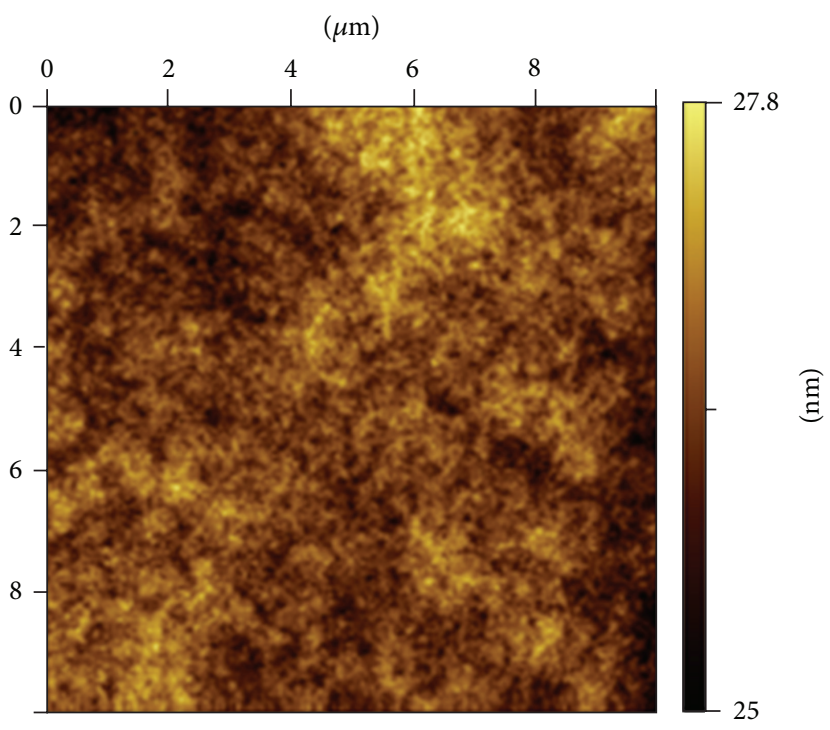

(a)

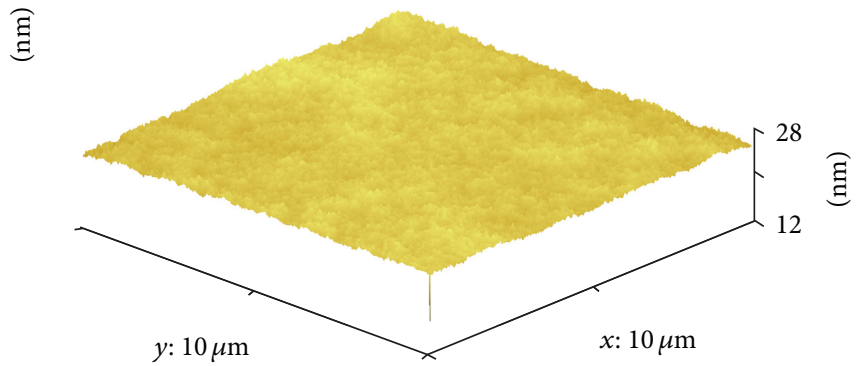

(b)

FIGURE 1: (a) Typical topographical AFM image, acquired in tapping mode, of the surface of a ITO/PANI/sol-gel substrate. The substrate was plasma cleaned for 10 seconds just prior to imaging. (b) Three-dimensional representation of (a).

and $81 \mathrm{~nm}( \pm 2.4, n=4)$ for the subsequently deposited solgel layer. For comparison, PANI adsorbed on an oxidized Si wafer produced a thickness of $2.9 \mathrm{~nm}( \pm 0.17, n=3)$, confirming that multilayer adsorption occurred on ITO. The water contact angle of the as-deposited sol-gel surface was $53.8^{\circ} \pm 0.6^{\circ}(n=3)$, indicative of moderate hydrophobicity that is attributed to the methyl groups of MTES [28]. Plasma cleaning for 10 seconds made the surface hydrophilic (water contact angle $<5^{\circ}$ ) which was compatible with fusion of a PSLB (see below). Representative AFM images of an ITO/PANI/sol-gel electrode are shown in Figure 1. The sol-gel surface was relatively smooth, with a RMS surface roughness of $0.7 \pm 0.35 \mathrm{~nm}(n=4)$, as compared to the underlying ITO surface (RMS roughness of $1.4-2 \mathrm{~nm}$ ) [28]. In addition to hydrophilicity, a relatively smooth surface promotes formation of a continuous PSLB [35].

The PANI layer is the electrochemically active portion of the ITO/PANI/sol-gel electrode (i.e., bare ITO does not exhibit any appreciable response to $\mathrm{pH}$ [25]). In previous work, we found that the acidic groups on the surface of ITO serve as ionizable counterions for adsorbed PANI [25]. Furthermore, the ITO surface acts as an acidic dopant that protonates the insulating emeraldine base (EB) form of PANI, rendering the adsorbed film electroactive and $\mathrm{pH}$-responsive in near-neutral $\mathrm{pH}$ conditions.

The response of several ITO/PANI/sol-gel electrodes to changes in the $\mathrm{pH}$ of the buffer in contact with the sol-gel layer was measured potentiometrically over the $\mathrm{pH}$ range of $4-9$, after soaking them overnight in $\mathrm{pH} 4$ buffer as a preconditioning step. Figure 2 shows a typical response curve. The response time, defined as the time required to reach a stable potential (drift of $<0.2 \mathrm{mV} /$ minute) after a step change in $\mathrm{pH}$, was about 1 minute. Over the $\mathrm{pH}$ range of 4-9, ITO/PANI/sol-gel electrodes displayed a pseudo-Nernstian,

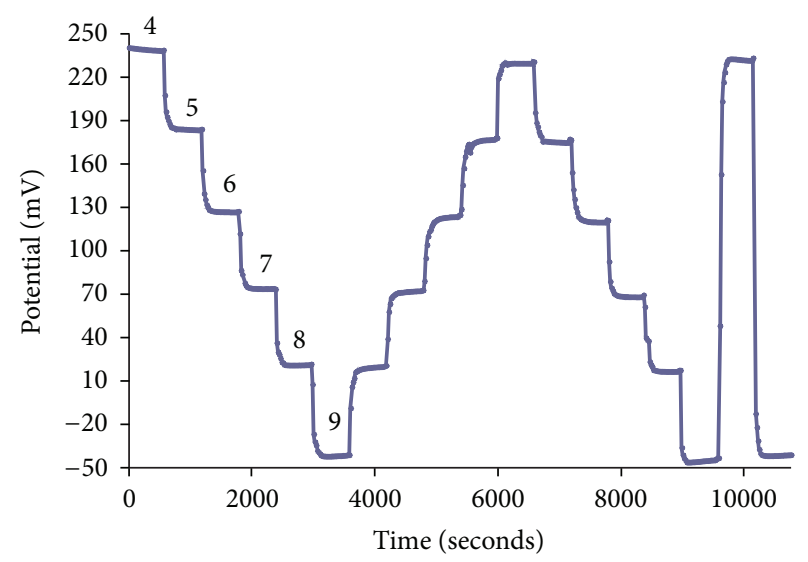

FIgure 2: Potential measured during step changes in the $\mathrm{pH}$ of the buffer in contact with an ITO/PANI/sol-gel sensor. After equilibration at $\mathrm{pH} 4$, the $\mathrm{pH}$ was incrementally increased to $\mathrm{pH} 9$, followed by cycles of increase and decrease. The numbers above each "plateau" during the initial increase denote the $\mathrm{pH}$. The sensor was equilibrated for 5 minutes before the $\mathrm{pH}$ was changed.

linear response with a slope of $52 \mathrm{mV} / \mathrm{pH}( \pm 2.7, n=4)$ as shown in Figure 3. The wide dynamic range of the $\mathrm{pH}$ response in Figure 3 is expected. PANI is a polyelectrolyte containing both amine and imine groups. The proximity of these groups in the polymer chains and differences in their local chemical environment (e.g., due to variations in chain packing in the pores) should produce a broad distribution of chemically inequivalent sites and a correspondingly broad response to changes in bulk $\mathrm{pH}[22,25,36]$.

This response time of $1 \mathrm{~min}$ is comparable to that of PANI nanowires grown electrochemically in the pores of 


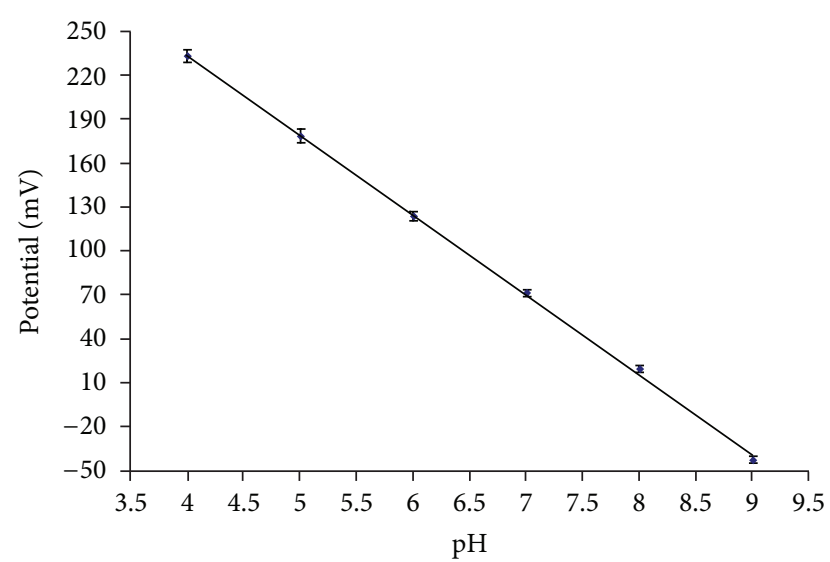

FIgURE 3: Potential measured as a function of $\mathrm{pH}$ of the buffer in contact with an ITO/PANI/sol-gel sensor. Measurements were made after an equilibration time of $5 \mathrm{~min}$ at each $\mathrm{pH}$. The slope of the plot was $52 \mathrm{mV} / \mathrm{pH}$ over the $\mathrm{pH}$ range of $4-9\left(R^{2}=0.9994\right)$. The error bars represent the standard deviation of three measurements.

a sol-gel glass layer coated on ITO [28]. However for the latter construct, the slope of the $\mathrm{pH}$ response curve was only $38 \mathrm{mV} / \mathrm{pH}$ [28]. This was attributed to the high number density of silanol groups in the pores which makes the solvent in the pores more acidic than the bulk solvent and buffers $\mathrm{pH}$ changes in the pores that result from changes in the bulk $\mathrm{pH}$ [37]. The significantly greater slope measured here for ITO/PANI/sol-gel electrodes is attributed the fact that the PANI and sol-gel layers are stratified rather than intercalated; thus the silanols have less effect on the response of the PANI layer to changes in bulk $\mathrm{pH}$. Support for this idea is provided by results from experiments on PANI/ITO electrodes lacking a sol-gel overlayer. Over the $\mathrm{pH}$ range of $4-9$, the response was linear with a slope of $52 \mathrm{mV} / \mathrm{pH}( \pm 5, n=2)$. This result is consistent with published data [25] and shows that the sol-gel layer did not measurably affect the $\mathrm{pH}$ sensitivity of the PANI layer.

Given that ITO is highly transparent in the visible spectrum, absorbance spectroscopy can also be used to monitor the $\mathrm{pH}$-dependent response of PANI films [22-25, 29]. Attenuated total reflection (ATR) spectra of ITO/PANI/solgel electrodes measured as a function of $\mathrm{pH}$ are shown in Supplementary Material. The data are consistent with previous ATR studies of the $\mathrm{pH}$-dependent equilibrium between the EB and the emeraldine salt (ES) forms of PANI [25] and demonstrate that both optical and electrochemical sensing methods can be combined in a complementary manner when the PANI film is supported on an optically transparent electrode [29].

\subsection{PSLB Deposition on ITO/PANI/Sol-Gel Electrodes.} Experiments were conducted to determine if a continuous PSLB could be deposited by vesicle fusion onto the upper surface of ITO/PANI/sol-gel electrodes. Epifluorescence images of PSLBs composed of NBD-PC/egg PC deposited on ITO/PANI/sol-gel electrodes were featureless, indicating that the bilayer was uniform on a length scale of several $\mathrm{mm}$

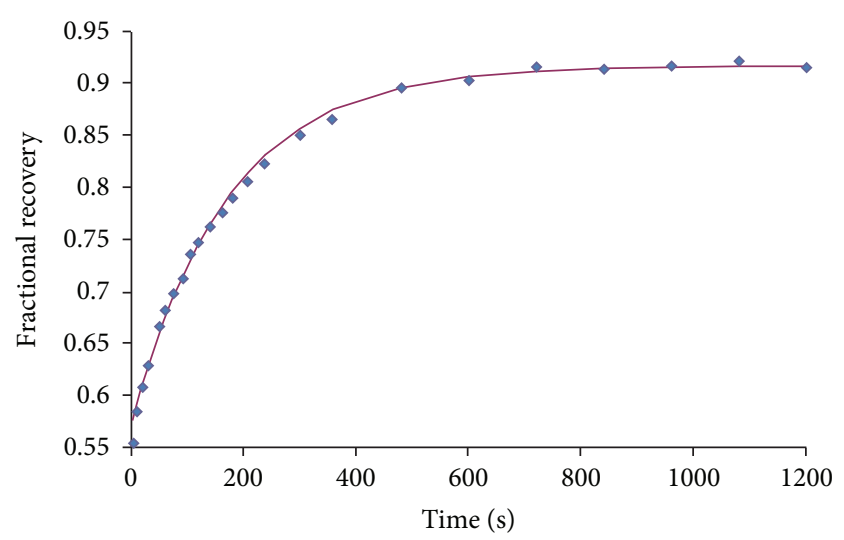

FIGURE 4: Typical FRAP curve showing recovery of bleached fluorescence in a PSLB composed of eggPC/NBD-PC $(95: 5 \mathrm{~mol} / \mathrm{mol})$ deposited on an ITO/PANI/sol-gel electrode.

and that unfused vesicles were largely removed by rinsing. PSLBs were also characterized by fluorescence recovery after photobleaching (FRAP) to assess bilayer fluidity. An example recovery curve is shown in Figure 4. The lateral diffusion coefficient $(D)$ was $2.2 \pm 0.5 \mu \mathrm{m}^{2} / \mathrm{s}(n=10)$ with a percent recovery of $91 \pm 2.1 \%(n=10)$. These values are consistent with previous measurements of lipid diffusion on sol-gel substrates [28]. They show that the lipid films are laterally continuous, and are not composed predominately of adsorbed vesicles (for which the percent recovery would be minimal) [38]. The percent recovery shows that about $10 \%$ of the bilayer is immobile on the timescale of the measurement, which is attributable to the greater surface roughness of the sol-gel surface relative to glass (on which the recovery is typically close to $100 \%)[28,39,40]$.

A continuous PSLB that is relatively free of defects should present a significant barrier to transmembrane diffusion of ions. The capability of PSLBs to attenuate the $\mathrm{pH}$ response of ITO/PANI/sol-gel electrodes was therefore investigated. In these experiments, the electrode potential was monitored as a function of time following a step change in the buffer $\mathrm{pH}$ above the PSLB. Data from a typical experiment is shown in Figure 5. Transmembrane diffusion of proton/hydroxyl ions was blocked by the PSLB as evidenced by a potential drift of less than $1 \mathrm{mV}$ per hour for 2.5 hours. After 2.5 hours, a slow increase of ca. $4 \mathrm{mV}$ per hour was observed. Slow dissipation of a small $\mathrm{pH}$ gradient is indicative of a membrane having a low density of ion-permeable defects, as described in further detail in [28]. After 4 hours, the bilayer was disrupted by introducing air bubbles into the cell. This caused a large increase in potential (about $10 \mathrm{mV}$ per minute), demonstrating that the minimal change in potential prior to 4 hours was due to the high ion impermeability of the PSLB.

\section{Conclusions}

A new type of optically transparent, planar $\mathrm{pH}$ electrode suitable as a substrate for a PSLB is described here. Previous constructs had significant drawbacks, specifically weak adhesion 


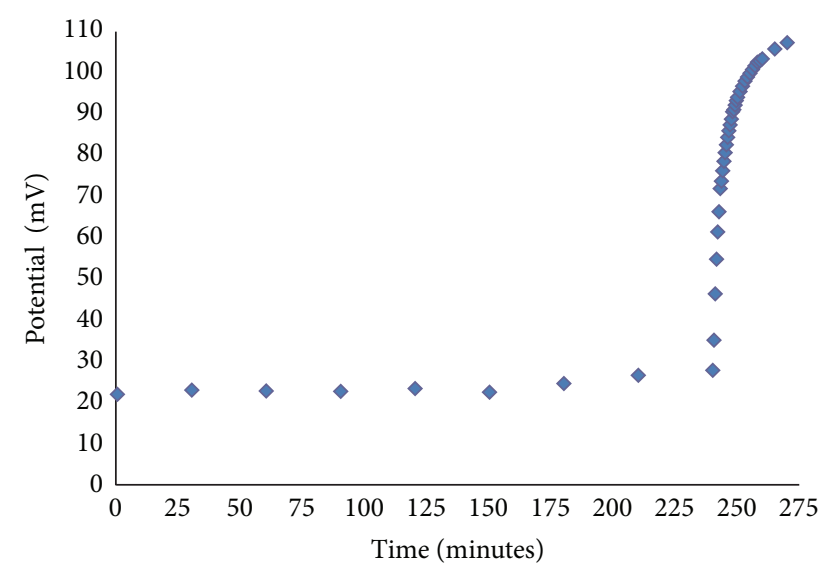

FIGURE 5: Potential of an ITO/PANI/sol-gel electrode coated with a PSLB. The coated electrode was equilibrated overnight at $\mathrm{pH} 8$. Ten minutes after initiating periodic measurements of the electrode potential, the $\mathrm{pH}$ of the buffer in contact with the PSLB was reduced to 6.18. The PSLB was physically disrupted at $t=240$ minutes by introducing air bubbles in contact with the PSLB.

of the PSLB [29] and more complex fabrication procedures [28]. In contrast, the new design is much simpler to fabricate than the PANI nanowire-based construct [28], and the upper sol-gel glass surface promotes vesicle fusion comparable to conventional glass and silica substrates. Moreover, the new design has a sensitivity of $52 \mathrm{mV} / \mathrm{pH}$ whereas that of the nanowire-based design was only $38 \mathrm{mV} / \mathrm{pH}$. The fluid PSLB on the sol-gel surface blocks the $\mathrm{pH}$ response due to the very low ion permeability of the membrane. Thus this new type of electrode should be useful for spectroelectrochemical monitoring of transmembrane proton transport via ionophores and ion channels.

\section{Acknowledgments}

During its initial stages, this work was partially supported by the National Science Foundation under Grant no. CHE0518702. The latter stages of this work were funded by the National Institutes of Health, National Institute of Biomedical Imaging and Bioengineering, under Grant no. R01EB007047. Any opinions, findings, and conclusions or recommendations expressed herein are those of the authors and do not necessarily reflect the views of the National Science Foundation, the National Institute of Biomedical Imaging and Bioengineering, or the National Institutes of Health. C. Ge gratefully acknowledges partial support from a fellowship from Merck Research Laboratories and a fellowship from the Proposition 301 Initiative on Photonics, Arizona Board of Regents.

\section{References}

[1] J. Goc, M. Hara, T. Tateishi, and J. Miyake, "Reconstructed lightharvesting system for photosynthetic reaction centres," Journal of Photochemistry and Photobiology A, vol. 93, no. 2-3, pp. 137144, 1996.
[2] D. Gust, T. A. Moore, and A. L. Moore, "Mimicking photosynthetic solar energy transduction," Accounts of Chemical Research, vol. 34, no. 1, pp. 40-48, 2001.

[3] B. A. Cornell, V. L. B. Braach-Maksvytis, L. G. King et al., "A biosensor that uses ion-channel switches," Nature, vol. 387, no. 6633, pp. 580-583, 1997.

[4] H. T. Tien, Z. Salamon, and A. Ottova, "Lipid bilayer-based sensors and biomolecular electronics," Critical Reviews in Biomedical Engineering, vol. 18, p. 323, 1991.

[5] T. J. M. Luo, R. Soong, E. Lan, B. Dunn, and C. Montemagno, "Photo-induced proton gradients and ATP biosynthesis produced by vesicles encapsulated in a silica matrix," Nature Materials, vol. 4, no. 3, pp. 220-224, 2005.

[6] M. Tanaka and E. Sackmann, "Polymer-supported membranes as models of the cell surface," Nature, vol. 437, no. 7059, pp. 656663, 2005.

[7] R. Naumann, E. K. Schmidt, A. Jonczyk et al., "The peptidetethered lipid membrane as a biomimetic system to incorporate cytochrome c oxidase in a functionally active form," Biosensors and Bioelectronics, vol. 14, no. 7, pp. 651-662, 1999.

[8] J. D. Burgess, M. C. Rhoten, and F. M. Hawkridge, "Cytochrome c oxidase immobilized in stable supported lipid bilayer membranes," Langmuir, vol. 14, no. 9, pp. 2467-2475, 1998.

[9] R. Naumann, T. Baumgart, P. Graber, A. Jonczyk, A. Offenhausser, and W. Knoll, "Proton transport through a peptidetethered bilayer lipid membrane by the $\mathrm{H}^{+}$-ATP synthase from chloroplasts measured by impedance spectroscopy," Biosensors and Bioelectronics, vol. 17, no. 1-2, pp. 25-34, 2002.

[10] L. J. C. Jeuken, R. J. Bushby, and S. D. Evans, "Proton transport into a tethered bilayer lipid membrane," Electrochemistry Communications, vol. 9, no. 4, pp. 610-614, 2007.

[11] I. K. Vockenroth, P. P. Atanasova, A. T. A. Jenkins, and I. Köper, "Incorporation of $\alpha$-hemolysin in different tethered bilayer lipid membrane architectures," Langmuir, vol. 24, no. 2, pp. 496-502, 2008.

[12] D. J. McGillivray, G. Valincius, F. Heinrich et al., "Structure of functional Staphylococcus aureus $\alpha$-hemolysin channels in tethered bilayer lipid membranes," Biophysical Journal, vol. 96, no. 4, pp. 1547-1553, 2009.

[13] E. K. Schmitt, M. Nurnabi, R. J. Bushby, and C. Steinem, "Electrically insulating pore-suspending membranes on highly ordered porous alumina obtained from vesicle spreading," Soft Matter, vol. 4, no. 2, pp. 250-253, 2008.

[14] R. Naumann, S. M. Schiller, F. Giess et al., "Tethered lipid bilayers on ultraflat gold surfaces," Langmuir, vol. 19, no. 13, pp. 5435-5443, 2003.

[15] R. Naumann, D. Walz, S. M. Schiller, and W. Knoll, "Kinetics of valinomycin-mediated $\mathrm{K}+$ ion transport through tethered bilayer lipid membranes," Journal of Electroanalytical Chemistry, vol. 550-551, pp. 241-252, 2003.

[16] H. Hillebrandt, G. Wiegand, M. Tanaka, and E. Sackmann, "High electric resistance polymer/lipid composite films on indium-tin-oxide electrodes," Langmuir, vol. 15, no. 24, pp. 8451-8459, 1999.

[17] V. Atanasov, N. Knorr, R. S. Duran et al., "Membrane on a chip: a functional tethered lipid bilayer membrane on silicon oxide surfaces," Biophysical Journal, vol. 89, no. 3, pp. 1780-1788, 2005.

[18] T. Skotheim, Handbook of Conducting Polymers, Marcel Dekker, New York, NY, USA, 1986.

[19] S. Y. Cui and S. M. Park, "Electrochemistry of conductive polymers XXIII: polyaniline growth studied by electrochemical 
quartz crystal microbalance measurements," Synthetic Metals, vol. 105, no. 2, pp. 91-98, 1999.

[20] W. S. Huang, B. D. Humphrey, and A. G. Macdiarmid, "Polyaniline, a novel conducting polymer. Morphology and chemistry of its oxidation and reduction in aqueous electrolytes," Journal of the Chemical Society, Faraday Transactions, vol. 82, pp. 23852400, 1986.

[21] T. Ohsaka, Y. Ohnuki, N. Oyama, G. Katagiri, and K. Kamisako, "IR absorption spectroscopic identification of electroactive and electroinactive polyaniline films prepared by the electrochemical polymerization of aniline," Journal of Electroanalytical Chemistry, vol. 161, no. 2, pp. 399-406, 1984.

[22] U. W. Grummt, A. Pron, M. Zagorska, and S. Lefrant, "Polyaniline based optical pH sensor," Analytica Chimica Acta, vol. 357, no. 3, pp. 253-259, 1997.

[23] E. Pringsheim, E. Terpetschnig, and O. S. Wolfbeis, "Optical sensing of $\mathrm{pH}$ using thin films of substituted polyanilines," Analytica Chimica Acta, vol. 357, no. 3, pp. 247-252, 1997.

[24] T. Lindfors and A. Ivaska, "pH sensitivity of polyaniline and its substituted derivatives," Journal of Electroanalytical Chemistry, vol. 531, no. 1, pp. 43-52, 2002.

[25] C. Ge, N. R. Armstrong, and S. S. Saavedra, "pH-sensing properties of poly(aniline) ultrathin films self-assembled on indium-tin oxide," Analytical Chemistry, vol. 79, no. 4, pp. 14011410, 2007.

[26] G. Cui, J. S. Lee, S. J. Kim, H. Nam, G. S. Cha, and H. D. Kim, "Potentiometric $\mathrm{pCO}_{2}$ sensor using polyaniline-coated pH-sensitive electrodes," Analyst, vol. 123, pp. 1855-1859, 1998.

[27] A. A. Karyakin, M. Vuki, L. V. Lukachova et al., "Processible polyaniline as an advanced potentiometric $\mathrm{pH}$ transducer. Application to biosensors," Analytical Chemistry, vol. 71, no. 13, pp. 2534-2540, 1999.

[28] C. Ge, K. S. Orosz, N. R. Armstrong, and S. S. Saavedra, "Poly(aniline) nanowires in sol-gel coated ITO: a pH-responsive substrate for planar supported lipid bilayers," ACS Applied Materials and Interfaces, vol. 3, no. 7, pp. 2677-2685, 2011.

[29] T. W. McBee, L. Wang, C. Ge et al., "Characterization of proton transport across a waveguide-supported lipid bilayer," Journal of the American Chemical Society, vol. 128, no. 7, pp. 2184-2185, 2006.

[30] E. Reimhult, F. Höök, and B. Kasemo, "Intact vesicle adsorption and supported biomembrane formation from vesicles in solution: influence of surface chemistry, vesicle size, temperature, and osmotic pressure," Langmuir, vol. 19, no. 5, pp. 1681-1691, 2003.

[31] W. J. Doherty, C. L. Donley, N. R. Armstrong, and S. S. Saavedra, "Broadband spectroelectrochemical attenuated total reflectance instrument for molecular adlayer studies," Applied Spectroscopy, vol. 56, no. 7, pp. 920-927, 2002.

[32] H. Zhang, K. S. Orosz, H. Takahashi, and S. Scott Saavedra, "Broadband plasmon waveguide resonance spectroscopy for probing biological thin films," Applied Spectroscopy, vol. 63, no. 9, pp. 1062-1067, 2009.

[33] P. J. Skrdla, S. S. Saavedra, N. R. Armstrong, S. B. Mendes, and N. Peyghambarian, "Sol-gel-based, planar waveguide sensor for water vapor," Analytical Chemistry, vol. 71, no. 7, pp. 1332-1337, 1999.

[34] L. Yang, S. S. Saavedra, and N. R. Armstrong, "Sol-gel-based, planar waveguide sensor for gaseous iodine," Analytical Chemistry, vol. 68, pp. 1834-1841, 1996.
[35] K. J. Seu, A. P. Pandey, F. Haque, E. A. Proctor, A. E. Ribbe, and J. S. Hovis, "Effect of surface treatment on diffusion and domain formation in supported lipid bilayers," Biophysical Journal, vol. 92, pp. 2445-2450, 2007.

[36] R. Vonklitzing and H. Mohwald, "Proton concentration profile in ultrathin polyelectrolyte films," Langmuir, vol. 11, pp. 35543559, 1995.

[37] B. Dunn and J. I. Zink, "Probes of pore environment and molecule-matrix interactions in sol-gel materials," Chemistry of Materials, vol. 9, no. 11, pp. 2280-2291, 1997.

[38] C. Hamai, T. Yang, S. Kataoka, P. S. Cremer, and S. M. Musser, "Effect of average phospholipid curvature on supported bilayer formation on glass by vesicle fusion," Biophysical Journal, vol. 90, no. 4, pp. 1241-1248, 2006.

[39] K. C. Weng, J. J. R. Stålgren, D. J. Duval, S. H. Risbud, and C. W. Frank, "Fluid biomembranes supported on nanoporous aerogel/xerogel substrates," Langmuir, vol. 20, no. 17, pp. 7232 7239, 2004.

[40] K. C. Weng, J. J. R. Stalgren, S. H. Risbud, and C. W. Frank, "Planar bilayer lipid membranes supported on mesoporous aerogels, xerogels, and Vycor glass: an epifluorescence microscopy study," Journal of Non-Crystalline Solids, vol. 350, pp. 46-53, 2004. 

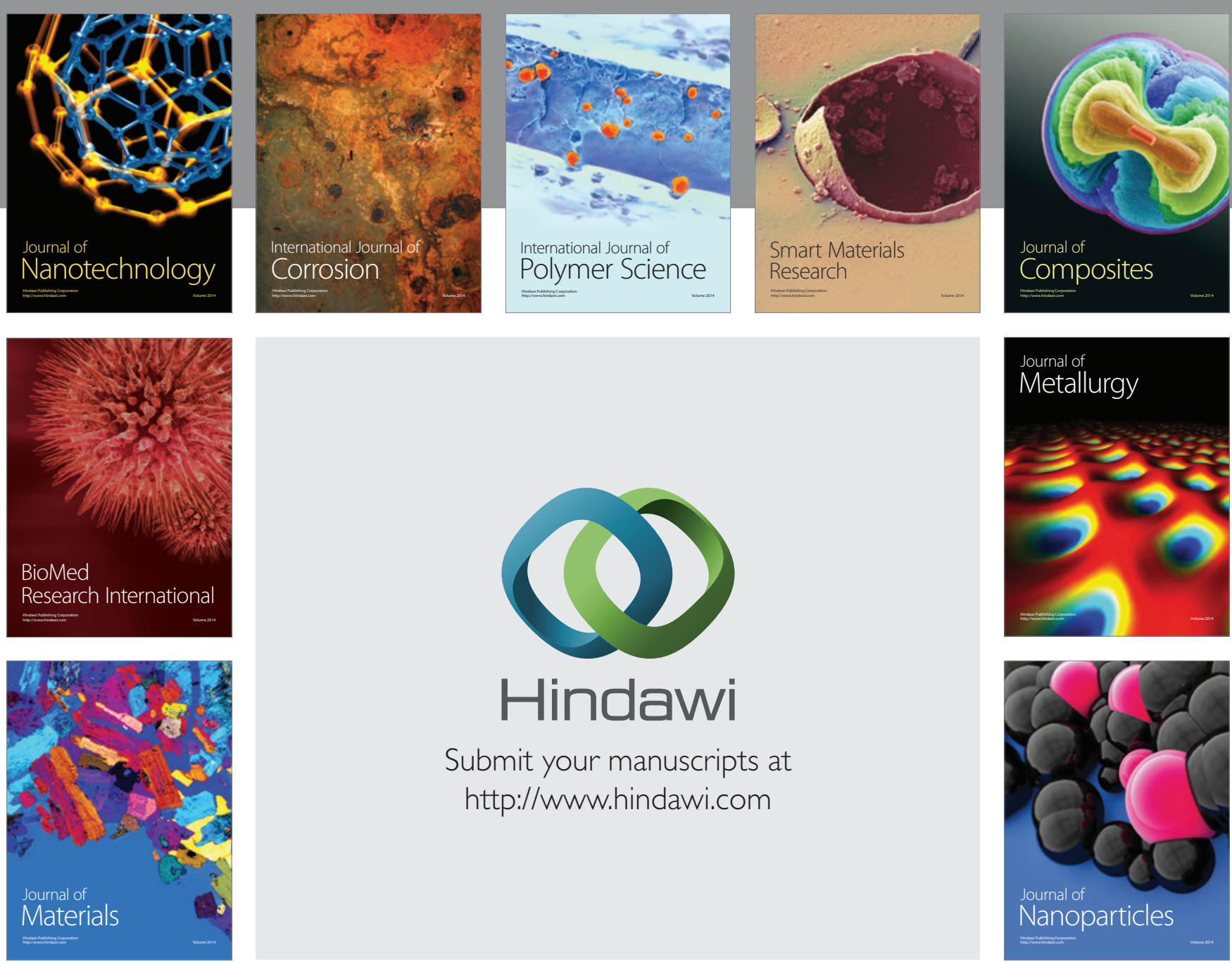

Submit your manuscripts at http://www.hindawi.com
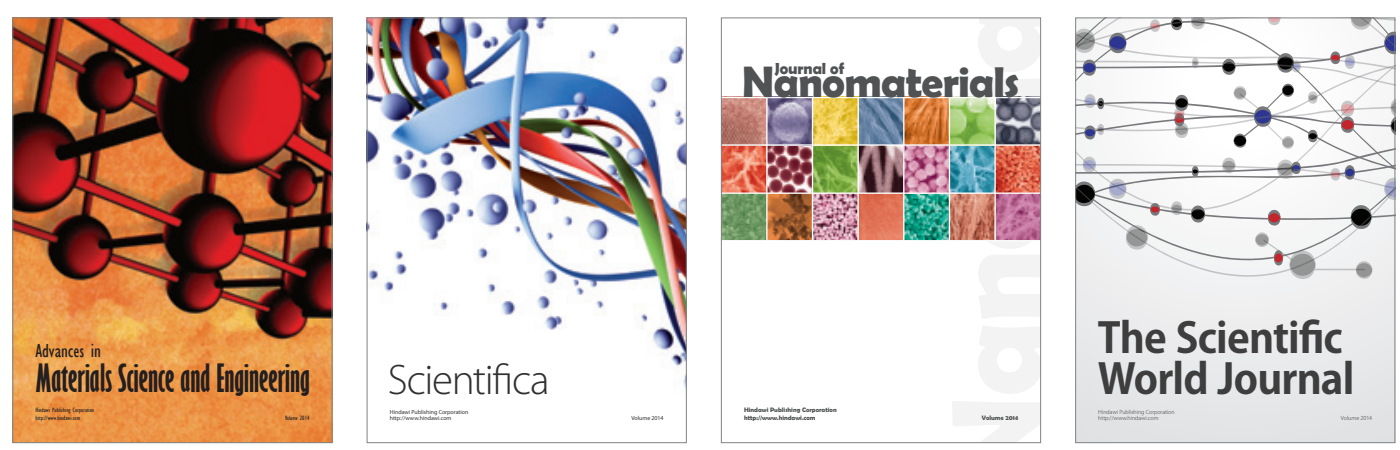

\section{The Scientific World Journal}
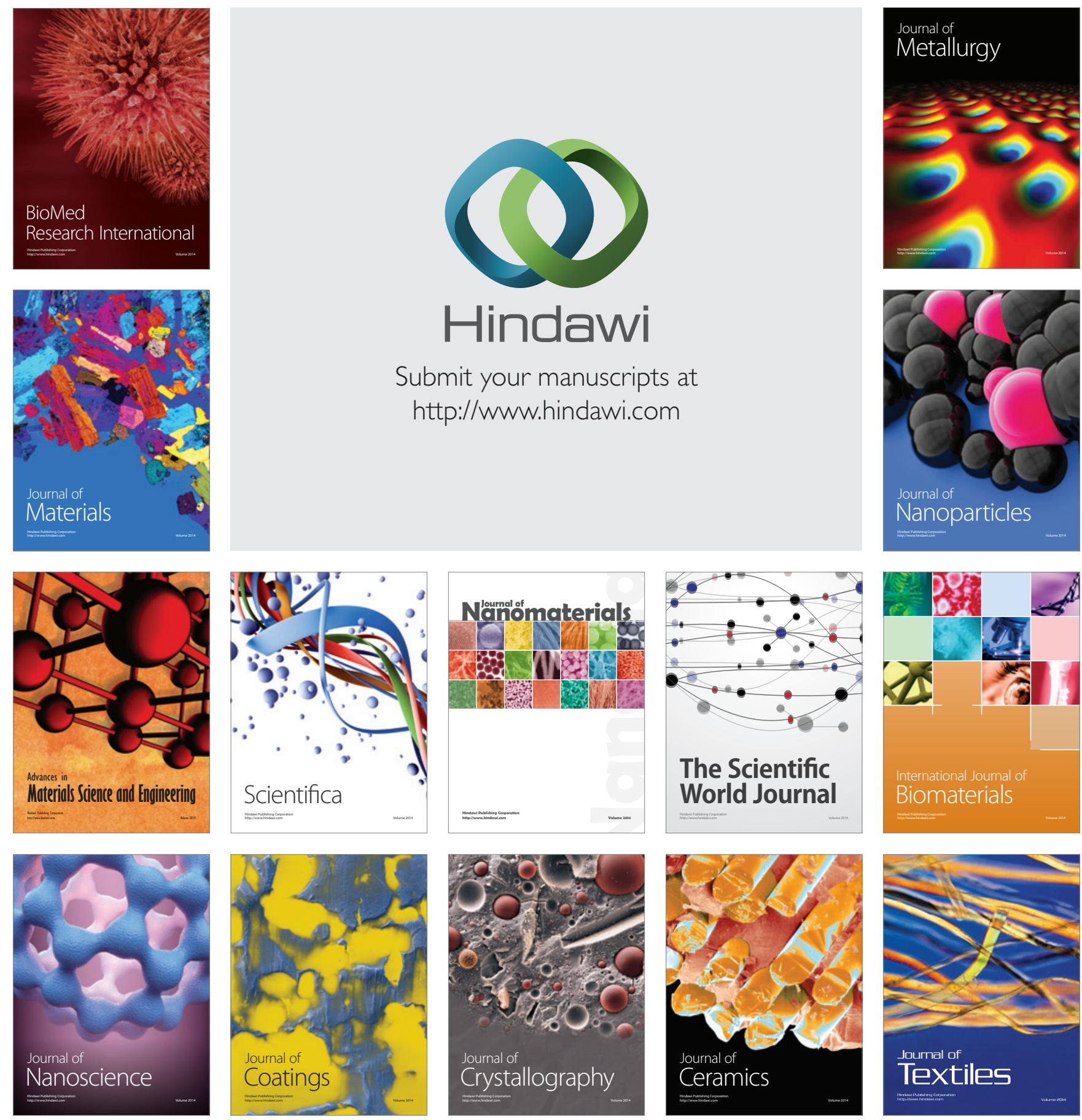